\title{
Pacific
}

Journal of

Mathematics

\section{ON MODULI OF INSTANTON BUNDLES ON $\mathbb{P}^{2 n+1}$}

Vincenzo ANCONA AND Giorgio Maria OtTAVIANi 


\title{
ON MODULI OF INSTANTON BUNDLES ON $\mathbb{P}^{2 n+1}$
}

\author{
VinCENZO ANCONA AND G. OttaViani
}

\begin{abstract}
Let $\mathrm{MI}_{\mathbb{P}^{2 n+1}}(k)$ be the moduli space of stable instanton bundles on $\mathbb{P}^{2 n+1}$ with $c_{2}=k$. We prove that $\mathrm{MI}_{\mathbb{P}^{2 n+1}}(2)$ is smooth, irreducible, unirational and has zero Euler-Poincaré characteristic, as it happens for $\mathbb{P}^{3}$. We find instead that $\mathrm{MI}_{\mathbb{P}^{5}}(3)$ and $\mathrm{MI}_{\mathbb{P}^{5}}(4)$ are singular.
\end{abstract}

\section{Definition and preliminaries.}

Instanton bundles on a projective space $\mathbb{P}^{2 n+1}(\mathbb{C})$ were introduced in $[$ OS and $[\mathbf{S T}]$. In $[\mathbf{A O}]$ we studied their stability, proving in particular that special symplectic instanton bundles on $\mathbb{P}^{2 n+1}$ are stable, and that on $\mathbb{P}^{5}$ every instanton bundle is stable.

In this paper we study some moduli spaces $\operatorname{MI}_{\mathbb{P}^{2 n+1}}(k)$ of stable instanton bundles on $\mathbb{P}^{2 n+1}$ with $c_{2}=k$. For $k=2$ we prove that $\mathrm{MI}_{\mathbb{P}^{2 n+1}}(2)$ is smooth, irreducible, unirational and has zero Euler-Poincaré characteristic (Theor. $3.2)$, just as in the case of $\mathbb{P}^{3}[\mathbf{H a r}]$.

We find instead that $\mathrm{MI}_{\mathbb{P}^{5}}(k)$ is singular for $k=3,4$ (theor. 3.3), which is not analogous with the case of $\mathbb{P}^{3}[\mathbf{E S}],[\mathbf{P}]$. To be more precise, all points corresponding to symplectic instanton bundles are singular. Theor. 3.3 gives, to the best of our knowledge, the first example of a singular moduli space of stable bundles on a projective space. The proof of Theorem 3.3 needs help from a personal computer in order to calculate the dimensions of some cohomology group [BaS].

We recall from $[\mathbf{O S}],[\mathbf{S T}]$ and $[\mathbf{A O}]$ the definition of instanton bundle on $\mathbb{P}^{2 n+1}(\mathbb{C})$.

Definition 1.1. A vector bundle $E$ of rank $2 n$ on $\mathbb{P}^{2 n+1}$ is called an instanton bundle of quantum number $k$ if

(i) The Chern polynomial is $c_{t}(E)=\left(1-t^{2}\right)^{-k}=1+k t^{2}+\left(\begin{array}{c}k+1 \\ 2\end{array}\right) t^{2}+\ldots$

(ii) $E(q)$ has natural cohomology in the range $-2 n-1 \leq q \leq 0$ (that is $h^{i}(E(q)) \neq 0$ for at most one $i=i(q)$ )

(iii) $\left.E\right|_{r} \simeq \mathcal{O}_{r}^{2 n}$ for a general line $r$.

Every instanton bundle is simple [AO]. There is the following characterization: 
Theorem 1.2 ([ST], [AO]). A vector bundle $E$ of rank $2 n$ on $\mathbb{P}^{2 n+1}$ satisfies the properties (i) and (ii) if and only if $E$ is the cohomology of a monad

$$
\mathcal{O}(-1)^{k} \stackrel{A}{\rightarrow} \mathcal{O}^{2 n+2 k} \stackrel{B}{\rightarrow} \mathcal{O}(1)^{k} .
$$

With respect to a fixed system of homogeneous coordinates the morphism $A$ (resp. $B$ ) of the monad can be identified with a $k \times(2 n+2 k$ ) (resp. $(2 n+2 k) \times k)$ matrix whose entries are homogeneous polynomials of degree 1. Then the conditions that (1.1) is a monad are equivalent to:

$A, B$ have rank $k$ at every point $x \in \mathbb{P}^{2 n+1}, A \cdot B=0$.

Definition 1.3. A bundle $S$ appearing in an exact sequence:

$$
0 \rightarrow S^{*} \rightarrow \mathcal{O}^{d} \stackrel{B}{\rightarrow} \mathcal{O}(1)^{c} \rightarrow 0
$$

is called a Schwarzenberger type bundle $(S T B)$.

The kernel bundle Ker $B$ in the monad (1.1) is the dual of a STB.

Definition 1.4. An instanton bundle is called special if it arises from a monad (1.1) where the morphism $B$ is defined in some system of homogeneous coordinates $\left(x_{0}, \ldots, x_{n}, y_{0}, \ldots, y_{n}\right)$ on $\mathbb{P}^{2 n+1}$ by the matrix

$$
B=\left[\begin{array}{ccc}
x_{0} & & \\
\vdots & \ddots & \\
x_{n} & & x_{0} \\
& \ddots & \vdots \\
& & x_{n} \\
y_{0} & & \\
\vdots & \ddots & \\
y_{n} & & y_{0} \\
& \ddots & \vdots \\
& & y_{n}
\end{array}\right]
$$


Example 1.5. Take

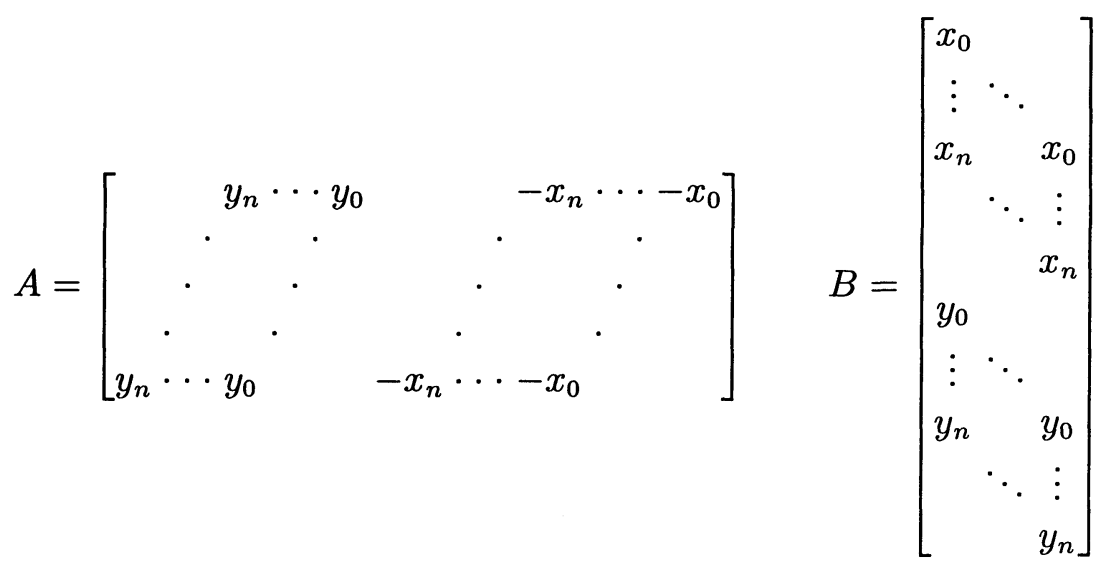

$E=\operatorname{Ker} B / \operatorname{Im} A$ is a special instanton bundle.

Property (iii) of the definition 1.1 can be checked by the following:

Theorem 1.6 [OS]. Let $E=\operatorname{Ker} B / \operatorname{Im} A$ as in (1.1). Let $r$ be the line joining two distinct points $P, Q \in \mathbb{P}^{2 n+1}$. Then

$$
\left.E\right|_{r} \simeq \mathcal{O}_{r}^{2 n} \Leftrightarrow A(P) \cdot B(Q) \quad \text { is an invertible matrix. }
$$

Example 1.7. Consider the special instanton bundle $E$ of the example 1.5. Let $P=(1,0, \ldots ; 0, \ldots, 0), Q=(0, \ldots, ; 0, \ldots, 1)$. Then

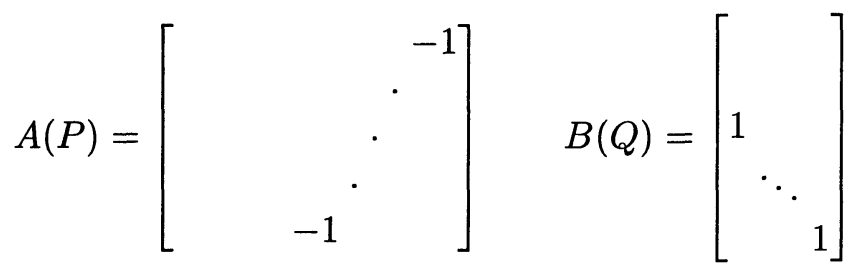

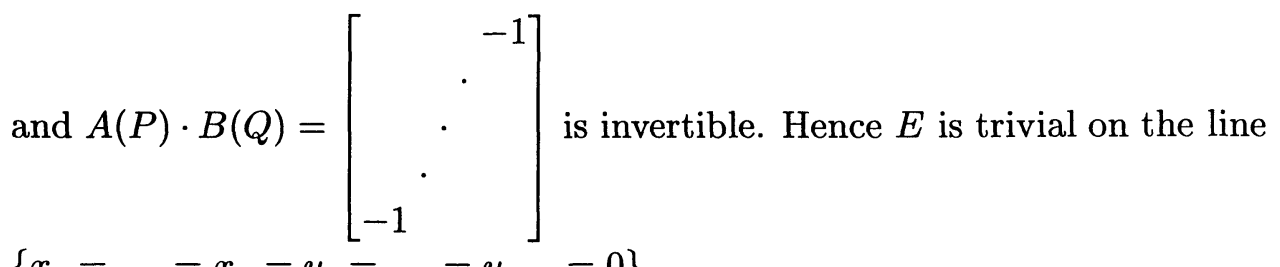
$\left\{x_{1}=\ldots=x_{n}=y_{0}=\ldots=y_{n-1}=0\right\}$.

Proposition 1.8. Let $E$ be an instanton bundle as in (1.1). Then $H^{2}\left(E \otimes E^{*}\right)=H^{2}\left[(\operatorname{Ker} B) \otimes\left(\operatorname{Ker} A^{t}\right)\right]$ 
Proof. See [AO] Theorem 3.13 and Remark 2.22.

Remark 1.9. If $E \simeq E^{*}$, then $H^{2}\left(E \otimes E^{*}\right)=H^{2}\left[\left(\operatorname{Ker} A^{t}\right) \otimes\left(\operatorname{Ker} A^{t}\right)\right]=H^{2}[(\operatorname{Ker} B) \otimes(\operatorname{Ker} B)]$.

Remark 1.10. The single complex associated with the double complex obtained by tensoring the two sequences

$$
\begin{aligned}
& 0 \rightarrow \operatorname{Ker} A^{t} \rightarrow \mathcal{O}^{2 n+2 k} \stackrel{A^{t}}{\rightarrow} \mathcal{O}(1)^{k} \rightarrow 0 \\
& 0 \rightarrow \operatorname{Ker} B^{t} \rightarrow \mathcal{O}^{2 n+2 k} \stackrel{B^{t}}{\rightarrow} \mathcal{O}(1)^{k} \rightarrow 0
\end{aligned}
$$

gives the resolution

$$
\begin{aligned}
0 \rightarrow\left(\operatorname{Ker} A^{t}\right) & \otimes(\operatorname{Ker} B) \rightarrow \mathcal{O}^{2 n+2 k} \otimes \mathcal{O}^{2 n+2 k} \\
& \rightarrow \mathcal{O}^{2 n+2 k} \otimes \mathcal{O}(1)^{k} \oplus \mathcal{O}(1)^{k} \otimes \mathcal{O}^{2 n+2 k} \stackrel{\alpha}{\rightarrow} \mathcal{O}(1)^{k} \otimes \mathcal{O}(1)^{k} \rightarrow 0
\end{aligned}
$$

where $\alpha=\left(A^{t} \otimes\right.$ id, id $\left.\otimes B\right)$.

Hence

$$
H^{2}\left(E \otimes E^{*}\right)=\text { Coker } H^{0}(\alpha)
$$

and its dimension can be computed using [BaS]. For the convenience of the reader we sketch the steps needed in the computations.

$A, B^{t}$ are given by $k \times(2 n+2 k)$ matrices whose entries are linear homogeneous polynomials.

$$
A \otimes \operatorname{Id}_{k}=\left(a_{1}, \ldots, a_{k(2 n+2 k)}\right)
$$

and

$$
\operatorname{Id}_{k} \otimes B^{t}=\left(b_{1}, \ldots, b_{k(2 n+2 k)}\right)
$$

are both $k^{2} \times(2 n+2 k) k$ matrices. Let

$$
C=\left(a_{1}, \ldots, a_{k(2 n+2 k)}, b_{1}, \ldots, b_{k(2 n+2 k)}\right) .
$$

We will denote by $\mathrm{syz}_{m} C$ the dimension of the space of the syzygies of $C$ of degree $m$. Then

$$
\begin{aligned}
h^{2}\left(E \otimes E^{*}\right) & =h^{0}\left(\mathcal{O}(2)^{k^{2}}\right)-(4 n+4 k) h^{0}\left(\mathcal{O}(1)^{k}\right)+\operatorname{syz}_{1} C \\
& =k(n+1)[k(2 n-5)-8 n]+\operatorname{syz}_{1} C \\
h^{1}\left(E \otimes E^{*}\right) & =h^{2}\left(E \otimes E^{*}\right)+1-k^{2}+8 n^{2} k-4 n^{2}+3 n k^{2}-2 n^{2} k^{2} \\
& =1-6 k^{2}-8 k n-4 n^{2}+\operatorname{syz}_{1} C .
\end{aligned}
$$


Note also that $h^{0}(E(1))=\operatorname{syz}_{1} B^{t}-k$ and $h^{0}\left(E^{*}(1)\right)=\operatorname{syz}_{1} A-k$.

Remark 1.11. In the same way we obtain

$$
\begin{gathered}
h^{1}\left(E \otimes E^{*}(-1)\right)=\mathrm{syz}_{0} C \\
h^{2}\left(E \otimes E^{*}(-1)\right)=2 k(n k-2 n-k)+\mathrm{syz}_{0} C .
\end{gathered}
$$

\section{Example on $\mathbb{P}^{5}$.}

Let $(a, b, c, d, e, f)$ be homogeneous coordinates in $\mathbb{P}^{5}$.

Example 2.1. $(k=3)$ Let

$$
\begin{aligned}
B^{t} & =\left[\begin{array}{cc}
a b c & d e f \\
a b b & d e f \\
a b c & d e f f
\end{array}\right] \\
A & =\left[\begin{array}{cc}
f e d & -c-b-a \\
f \text { e d } & -c-b-a \\
f \text { e d } & -c-b-a
\end{array}\right] .
\end{aligned}
$$

The corresponding monad gives a special symplectic instanton bundle on $\mathbb{P}^{5}$ with $k=3$. With the notation of remark 1.10 , using [BaS] we can compute syz $_{0} C=14, \mathrm{syz}_{1} C=174$. Hence $h^{2}\left(E \otimes E^{*}\right)=3$ from the formulas of Remark 1.10. Moreover $h^{0}(E(1))=4$.

Example 2.2. $\quad(k=3)$ Let $B^{t}$ as in the Example 2.1 and

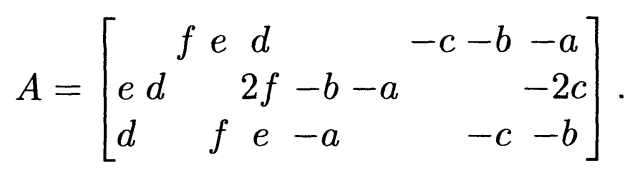

We have syz $0=10, \operatorname{syz}_{1} C=171$. Hence $h^{2}\left(E \otimes E^{*}\right)=0$. We can compute also the syzygies of $B^{t}$ and $A$ and we get $h^{0}(E(1))=4, h^{0}\left(E^{*}(1)\right)=3$, hence $E$ is not self-dual.

Example 2.3. $(k=4)$ Let

$$
\begin{gathered}
B^{t}=\left[\begin{array}{cc}
a b c & d e f \\
a b c & d e f \\
a b c & d e f \\
a b c & d e f
\end{array}\right] \\
A=\left[\begin{array}{cc}
f e d & -c-b-a \\
f e d & -c-b-a \\
f e d & -c-b-a
\end{array}\right]
\end{gathered}
$$


$E$ is a special symplectic instanton bundle with $k=4$. We compute

$$
h^{2}\left(E \otimes E^{*}\right)=12
$$

Example 2.4. $(k=4)$ Let $B^{t}$ as in the Example 2.3. Let

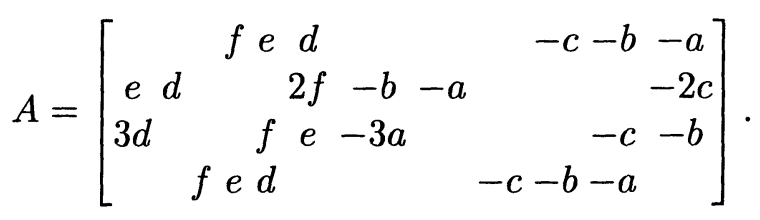

In this case $h^{2}\left(E \otimes E^{*}\right)=6, h^{0}(E(1))=4, h^{0}\left(E^{*}(1)\right)=3$.

Example 2.5. $(k=4)$ Let $B^{t}$ as in the Example 2.3. Let

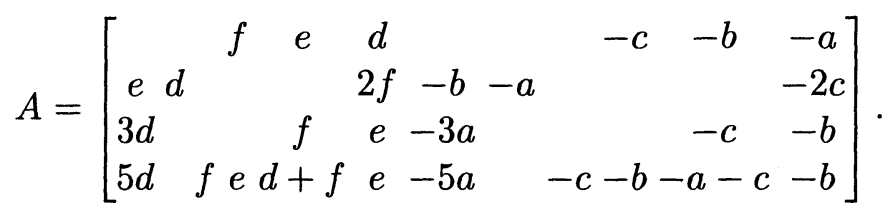

Now $H^{2}\left(E \otimes E^{*}\right)=0, h^{0}(E(1))=4, h^{0}\left(E^{*}(1)\right)=2$.

\section{On the singularities of moduli spaces.}

The stable Schwarzenberger type bundles on $\mathbb{P}^{m}$ (see (1.2)) form a Zariski open subset of the moduli space of stable bundles. Let $N_{\mathbb{P}^{m}}(k, q)$ be the moduli space of stable STB whose first Chern class is $k$ and whose rank is $q$. The following proposition is easy and well known:

Proposition 3.1. The space $N_{\mathbb{P} m}(k, q)$ is smooth, irreducible of dimension $1-k^{2}-(q+k)^{2}+k(q+k)(m+1)$.

We denote by $\mathrm{MI}_{\mathbb{P}^{2 n+1}}(k)$ the moduli space of stable instanton bundles with quantum number $k$. It is an open subset of the moduli space of stable $2 n$-bundles on $\mathbb{P}^{2 n+1}$ with Chern polynomial $\left(1-t^{2}\right)^{-k}$.

On $\mathbb{P}^{5}$ (as on $\mathbb{P}^{3}$ ) all instanton bundles are stable by [AO], Theorem 3.6. $\mathrm{MI}_{\mathbb{P}^{2 n+1}}(2)$ is smooth ([AO] Theorem 3.14), unirational of dimension $4 n^{2}+12 n-3$ and has zero Euler-Poincaré characteristic ([BE], $\left.[\mathbf{K}]\right)$.

Theorem 3.2. The space $\mathrm{MI}_{\mathbb{P}^{2 n+1}}(2)$ is irreducible.

Proof. The moduli space $N=N_{\mathbb{P}^{2 n+1}}(2, n+2)$ of stable STB of rank $2 n+2$ and $c_{1}=2$ is irreducible of dimension $4 n^{2}+8 n-3$ by Prop. 3.1 
For a given instanton bundle $E$ there is a STB $S$ associated with $E$, which is stable ([AO], Theorem 2.8) and unique (ibid., Prop. 2.17). It is easy to prove that the map $\pi: M \rightarrow N$ defined by $\pi([E])=[S]$ is algebraic, moreover $\pi$ is dominant by [ST]. If $m=[E] \in M$, the fiber $\pi^{-1}(\pi(m))$ is a Zariski open subset of the grassmannian of planes in the vector space $H^{0}\left(\mathbb{P}^{2 n+1}, S^{*}(1)\right)$, where $\pi(m)=[S]$; by the Theorem 3.14 of $[\mathbf{A O}], h^{0}\left(\mathbb{P}^{2 n+1}, S^{*}(1)\right)=2 n+2$, hence $\operatorname{dim} \pi^{-1}(\pi(m))=4 n$.

In order to prove that $M$ is irreducible, we suppose by contradiction that there are at least two irreducible components $M_{0}$ and $M_{1}$ of $M$. Then $M_{0} \cap M_{1}=\emptyset(M$ is smooth $), \pi\left(M_{0}\right)$ and $\pi\left(M_{1}\right)$ are constructible subset of $N$ by Chevalley's theorem. Looking at the dimensions of $M_{0}, M_{1}, N$ and the fibers of $\pi$ we conclude that both $\pi\left(M_{0}\right)$ and $\pi\left(M_{1}\right)$ must contain an open subset of $N$, which implies $\pi\left(M_{0}\right) \cap \pi\left(M_{1}\right) \neq \emptyset$ by the irreducibility of $N$. This is a contradiction because the fibers of $\pi$ are connected.

For $n \geq 2$ and $k \geq 3$, it is no longer true that $\operatorname{MI}_{\mathbb{P}^{2 n+1}}(k)$ is smooth. In fact on $\mathbb{P}^{5}$ we have:

Theorem 3.3. The space $\mathrm{MI}_{\mathbb{P}^{5}}(k)$ is singular for $k=3,4$. To be more precise, the irreducible component $M_{0}(k)$ of $\mathrm{MI}_{\mathbb{P}^{5}}(k)$ containing the special instanton bundles is generically reduced of dimension $54(k=3)$ or $65(k=4)$, and $\operatorname{MI}_{\mathbb{P}^{5}}(k)$ is singular at the points corresponding to special symplectic instanton bundles.

Proof. Let $E_{0}$ be the special instanton bundle on $\mathbb{P}^{5}$ of the Example 2.2( $k=$ 3 ) or of the Example $2.5(k=4)$. Then $h^{2}\left(E_{0} \otimes E_{0}^{*}\right)=0$ and $M_{0}(k)$ is smooth at the point corresponding to $E_{0}$, of dimension $h^{1}\left(E_{0} \otimes E_{0}^{*}\right)=54(k=3)$ or $65(k=4)$. In particular, $M_{0}(k)$ is generically reduced. If $E_{1}$ is a special symplectic instanton bundle on $\mathbb{P}^{5}$, the computations in 2.1 and 2.3 show that $h^{2}\left(E_{1} \otimes E_{1}^{*}\right)=3(k=3)$ or $12(k=4)$, and $h^{1}\left(E_{1} \otimes E_{1}^{*}\right)=57$ or 77 respectively. Hence $\operatorname{MI}_{\mathbb{P}^{5}}(k)$ is singular at $E_{1}$ for $k=3$ and 4 .

Remark 3.4. It is natural to conjecture that $\operatorname{MI}_{\mathbb{P}^{2 n+1}}(k)$ is singular for all $n \geq 2$ and $k \geq 3$.

Theorem 3.5. Let $E$ be an instanton bundle on $\mathbb{P}^{2 n+1}$ with $c_{2}(E)=k$. Then

$$
h^{1}(E(t))=0 \text { for } t \leq-2 \text { and } k-1 \leq t .
$$

Proof. The result is obvious for $t \leq-2$. It is sufficient to prove $h^{1}\left(S^{*}(t)\right)=0$ for $t \geq k-1$. We have

$$
S^{*}(t)=\bigwedge^{2 n+k-1} S(t-k)
$$


Taking wedge products of (1.2) we have the exact sequence

$$
\begin{aligned}
0 \rightarrow \mathcal{O}(t+1-2 n-2 k)^{\alpha_{0}} \rightarrow & \ldots \rightarrow \mathcal{O}(t-k-1)^{\alpha_{2 n+k-2}} \\
& \rightarrow \mathcal{O}(t-k)^{\alpha_{2 n+k-1}} \rightarrow \bigwedge^{2 n+k-1} S(t-k) \rightarrow 0
\end{aligned}
$$

for suitable $\alpha_{i} \in \mathbb{N}$ and from this sequence we can conclude.

Ellia proves Theorem 3.5 in the case of $\mathbb{P}^{3}$ ([E], Prop. IV.1). He also remarks that the given bound is sharp. This holds on $\mathbb{P}^{2 n+1}$ as it is shown by the following theorem, which points out that the special symplectic instanton bundles are the "furthest" from having natural cohomology.

Theorem 3.6. Let $E$ be a special symplectic instanton bundle on $\mathbb{P}^{2 n+1}$ with $c_{2}=k$. Then

$$
h^{1}(E(t)) \neq 0 \text { for }-1 \leq t \leq k-2 \text {. }
$$

Proof. For $n=1$ the thesis is immediate from the exact sequence

$$
0 \rightarrow \mathcal{O}(t-1) \rightarrow E(t) \rightarrow \mathcal{J}_{C}(t+1) \rightarrow 0
$$

where $C$ is the union of $k+1$ disjoint lines in a smooth quadric surface. Then the result follows by induction on $n$ by considering the sequence

$$
\left.0 \rightarrow E(t-2) \rightarrow E(t-1)^{2} \rightarrow E(t) \rightarrow E(t)\right|_{\mathbb{P}^{2 n-1}} \rightarrow 0
$$

and the fact that, for a particular choice of the subspace $\mathbb{P}^{2 n-1}$, the restriction $\left.E\right|_{\mathbb{P}^{2 n-1}}$ splits as the direct sum of a rank-2 trivial bundle and a special symplectic instanton bundle on $\mathbb{P}^{2 n-1}([\mathrm{ST}] 5.9)$.

Remark 3.7. In [OT] it is proved that if $E_{k}$ is a special symplectic

\begin{tabular}{|c|c|c|c|c|}
\hline \multicolumn{4}{|c|}{$h^{1}\left(E_{k} \otimes E_{k}^{*}\right) h^{2}\left(E_{k} \otimes E_{k}^{*}\right) \operatorname{dim} M_{0}(k)$} & $\mathrm{MI}_{\mathbb{P}^{5}}(k)$ \\
\hline$k=1$ & 14 & 0 & 14 & open subset of $\mathbb{P}^{14}$ \\
\hline$k=2$ & 37 & 0 & 37 & smooth, irreduc., unirat. \\
\hline$k=3$ & 57 & 3 & 54 & singular \\
\hline$k=4$ & 77 & 12 & 65 & singular \\
\hline$k \geq 2$ & $20 k-3$ & $3(k-2)^{2}$ & $?$ & $?$ \\
\hline
\end{tabular}
instanton bundle on $\mathbb{P}^{5}$ with $c_{2}=k$ then $h^{1}$ (End $E_{k}$ ) $=20 k-3$.

In the following table we summarize what we know about the component $M_{0}(k) \subset \operatorname{MI}_{\mathbb{P}^{5}}(k)$ containing $E_{k}$.

Table 3.10 


\section{References}

[AO] V. Ancona and G. Ottaviani, On the stability of special instanton bundles on $\mathbb{P}^{2 n+1}$, Trans. Amer. Math. Soc., 341 (1994), 677-693.

[BE] J. Bertin and G. Elencwajg, Symétries des fibrés vectoriels sur $\mathbb{P}^{n}$ et nombre d'Euler, Duke Math. J., 49 (1982), 807-831.

[BaS] D. Bayer and M. Stillman, Macaulay, a computer algebra system for algebraic geometry.

[BoS] G. Bohnhorst and H. Spindler, The stability of certain vector bundles on $\mathbb{P}^{n}$, Proc. Bayreuth Conference "Complex Algebraic Varieties", LNM 1507, Springer Berlin (1992), 39-50.

[E] Ph. Ellia, Some vanishings for the cohomology of stable rank two vector bundles on $\mathbb{P}^{3}$, J. reine angew. Math., 451 (1994), 1-14.

[ES] G. Ellingsrud and S.A. Stromme, Stable rank-2 bundles on $\mathbb{P}^{3}$ with $c_{1}=0$ and $c_{2}=3$, Math. Ann., 255 (1981), 123-137.

[Har] R. Hartshorne, Stable vector bundles of rank 2 on $\mathbb{P}^{3}$, Math. Ann., 238 (1978), 229-280.

[K] T. Kaneyama, Torus-equivariant vector bundles on projective spaces, Nagoya Math. J., 111 (1988), 25-40.

[M] M. Maruyama, Moduli of stable sheaves, II, J. Math. Kyoto Univ., 18 (1978), 557-614.

[OS] C. Okonek and H. Spindler, Mathematical instanton bundles on $\mathbb{P}^{2 n+1}$, Journal reine angew. Math., 364 (1986), 35-50.

[OT] G. Ottaviani and G. Trautmann, The tangent space at a special symplectic instanton bundle on $\mathbb{P}^{2 n+1}$, Manuscr. Math., 85 (1994), 97-107.

[P] J. LePotier, Sur l'espace de modules des fibrés de Yang et Mills, in Mathématique et Physique, Sém. E.N.S. 1979-1982, Basel-Stuttgart-Boston 1983.

[S] R.L.E. Schwarzenberger, Vector bundles on the projective plane, Proc. London Math. Soc., 11 (1961), 623-640.

[ST] H. Spindler and G. Trautmann, Special instanton bundles on $\mathbb{P}^{2 n+1}$, their geometry and their moduli, Math. Ann., 286 (1990), 559-592.

Received January 5, 1993. Both authors were supported by MURST and by GNSAGA of CNR.

Dipartimento di Matematica

Viale Morgagni $67 \mathrm{~A}$

I-50134 FIRENZE

E-mail address: ancona@udininw.math.unifi.it

AND

Dipartimento di Matematica

Via Vetoio, Coppito

I-67010 L'AQUILA

E-mail address: ottaviani@vxscaq.aquila.infn.it

Added in proof. After this paper has been written we received a preprint of R. MiróRoig and J. Orus-Lacort where they prove that the conjecture stated in the Remark 3.4 



\title{
PACIFIC JOURNAL OF MATHEMATICS
}

Founded by E. F. Beckenbach (1906-1982) and F. Wolf (1904-1989)

\section{EDITORS}

\author{
Sun-Yung Alice Chang (Managing Editor) \\ University of California \\ Los Angeles, CA 90095-1555 \\ pacific@math.ucla.edu
}

\section{F. Michael Christ}

University of California

Los Angeles, CA 90095-1555

christ@math.ucla.edu

Thomas Enright

University of California

San Diego, La Jolla, CA 92093

tenright@ucsd.edu

Nicholas Ercolani

University of Arizona

Tucson, AZ 85721

ercolani@math.arizona.edu
Robert Finn

Stanford University

Stanford, CA 94305

finn@gauss.stanford.edu

Vaughan F. R. Jones

University of California

Berkeley, CA 94720

vfr@math.berkeley.edu

Steven Kerckhoff

Stanford University

Stanford, CA 94305

spk@gauss.stanford.edu
Martin Scharlemann

University of California

Santa Barbara, CA 93106

mgscharl@math.ucsb.edu

Gang Tian

Courant Institute

New York University

New York, NY 10012-1100

tiang@taotao.cims.nyu.edu

V. S. Varadarajan

University of California

Los Angeles, CA 90095-1555

vsv@math.ucla.edu

\section{SUPPORTING INSTITUTIONS}

\section{CALIFORNIA INSTITUTE OF TECHNOLOGY \\ NEW MEXICO STATE UNIVERSITY \\ OREGON STATE UNIVERSITY \\ STANFORD UNIVERSITY \\ UNIVERSITY OF ARIZONA \\ UNIVERSITY OF BRITISH COLUMBIA \\ UNIVERSITY OF CALIFORNIA UNIVERSITY OF HAWAII}

\author{
UNIVERSITY OF MONTANA \\ UNIVERSITY OF NEVADA, RENO \\ UNIVERSITY OF OREGON \\ UNIVERSITY OF SOUTHERN CALIFORNIA \\ UNIVERSITY OF UTAH \\ UNIVERSITY OF WASHINGTON \\ WASHINGTON STATE UNIVERSITY
}

The supporting Institutions listed above contribute to the cost of publication of this Journal, but they are not owners or publishers and have no responsibility for its contents or policies.

Manuscripts must be prepared in accordance with the instructions provided on the inside back cover.

The Pacific Journal of Mathematics (ISSN 0030-8730) is published monthly except for July and August. Regular subscription rate: $\$ 215.00$ a year (10 issues). Special rate: $\$ 108.00$ a year to individual members of supporting institutions.

Subscriptions, orders for back issues published within the last three years, and changes of subscribers address should be sent to Pacific Journal of Mathematics, P.O. Box 4163, Berkeley, CA 94704-0163, U.S.A. Prior back issues are obtainable from Kraus Periodicals Co., Route 100, Millwood, NY 10546.

The Pacific Journal of Mathematics at the University of California, c/o Department of Mathematics, 981 Evans Hall, Berkeley, CA 94720 (ISSN 0030-8730) is published monthly except for July and August. Second-class postage paid at Berkeley, CA 94704, and additional mailing offices. POSTMASTER: send address changes to Pacific Journal of Mathematics, P.O. Box 6143, Berkeley, CA 94704-0163.

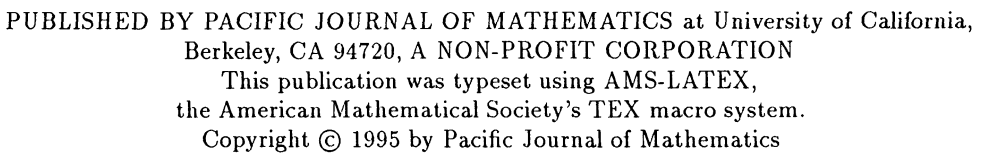




\section{PACIFIC JOURNAL OF MATHEMATICS}

\section{Volume $171 \quad$ No. $2 \quad$ December 1995}

On $H^{p}$-solutions of the Bezout equation

ERIC AMAR, JOAQUIM BRUNA FLORIS and ARTUR NiCOLAU

Amenable correspondences and approximation properties for von Neumann algebras

309

Claire ANANTHARAman-DElaroche

On moduli of instanton bundles on $\mathbb{P}^{2 n+1}$

VinCENZO ANCONA and Giorgio MaRia OtTAVIANI

Minimal surfaces with catenoid ends

JORGEN BERGLUND and WAYNE ROSSMAN

Permutation model for semi-circular systems and quantum random walks

PHILIPPE BIANE

The Neumann problem on Lipschitz domains in Hardy spaces of order less than one

RUSSELL M. BROWN

Matching theorems for twisted orbital integrals

409

REBECCA A. HERB

Uniform algebras generated by holomorphic and pluriharmonic functions on strictly

pseudoconvex domains

ALEXANDER IzZO

Quantum Weyl algebras and deformations of $U(g)$

NAIHUAN JING and JAMES ZHANG

Calcul du nombre de classes des corps de nombres

STÉPHANE LOUBOUTIN

On geometric properties of harmonic Lip ${ }_{1}$-capacity

PertTi MatTila and P. V. PARAmonov

Reproducing kernels and composition series for spaces of vector-valued holomorphic functions

Bent ØRsted and Genkai Zhang

Iterated loop modules and a filtration for vertex representation of toroidal Lie algebras

S. ESWARA RAO

The intrinsic mountain pass

MARTIN SCHECHTER

A Frobenius problem on the knot space

RON G. WANG

On complete metrics of nonnegative curvature on 2-plane bundles

DAVID YANG

Correction to: "Free Banach-Lie algebras, couniversal Banach-Lie groups, and more"

VLADIMIR G. PESTOV

Correction to: "Asymptotic radial symmetry for solutions of $\Delta u+e^{u}=0$ in a punctured disc"

KAI Seng (KAISing) CHOU (Tso) and Tom YAU-Heng WAN 